Pacific Journal of Mathematics

THE EXISTENCE OF DISCONTINUOUS MODULE 


\title{
THE EXISTENCE OF DISCONTINUOUS MODULE DERIVATIONS
}

\author{
NICHOLAS P. JEWELL
}

In this paper it is shown that if a commutative Banach algebra $B$ with identity has a maximal ideal $M$ whose algebraic powers $M^{2}, M^{3}, \cdots$ form a descending chain of ideals which never becomes constant then there exists a discontinuous module derivation from $B$ into a Banach- $B$ bimodule. This fact is linked with the known sufficient conditions for every module derivation from $B$ to be continuous when $B$ is separable. Some examples are given to demonstrate unusual behaviour in such chains of ideals in particular situations.

1. Introduction. Let $B$ be a Banach algebra over the complex field and let $X$ be a Banach-B-bimodule. We say that a linear mapping $D: B \rightarrow X$ is a module derivation if $D(a b)=a \cdot D(b)+D(a) \cdot b$ for $a, b$ in $B$ where * denotes the module operation. Recently some attention has been given to the problem of finding sufficient conditions on $B$ so that every module derivation from $B$ to any Banach$B$-bimodule is continuous. Two sufficient conditions for the case when $B$ is commutative and separable were described in [12] where it was noted that one of the conditions was also necessary for the automatic continuity of module derivations. In this paper we examine the extent to which the other condition is also necessary, proving that this is so under certain extra conditions on the maximal ideals of $B$. A related problem is to investigate the continuity of homomorphisms from $B$ into some Banach algebra. This has been investigated in the general situation most recently by W. G. Bade and P. C. Curtis, Jr. [1]. Notice that the existence of a discontinuous module derivation from $B$ into a Banach- $B$-bimodule implies the existence of a discontinuous homomorphism from $B$ into some Banach algebra [20, p. 49]. We make no attempt to discuss the particular case, $B=C(\Omega)$, where $\Omega$ is an infinite compact Hausdorff space (all module derivations are continuous in this situation-see [12]), where the recent spectacular work of H. G. Dales [5] and J. Esterle [8] has shown that, assuming the continuum hypothesis, there exists a discontinuous homomorphism from $C(\Omega)$ into a Banach algebra. It follows from their work that, when $B$ is an infinite dimensional commutative separable Banach algebra, there is a discontinuous homomorphism from $B$ into some Banach algebra [6,9] and it seems likely that the same result is true even when $B$ is not separable. 
Part of the significance of being able to construct a discontinuous module derivation $D$ on a commutative Banach algebra $A$ lies in the fact that when $D$ vanishes on a dense subset of $A$ it can be used to construct a commutative Banach algebra $B$ with two inequivalent complete norm topologies (see [15]). The author knows of no example of a commutative Banach algebra with non-unique complete norm topology which cannot be constructed in the fashion described in [15]. The existence of a discontinuous module derivation can also be used to construct Banach algebras on which there exist discontinuous algebra derivations. The author knows of no other way to construct discontinuous algebra derivations.

I would like to thank A. M. Davie for several stimulating conversations and also H. G. Dales for some helpful correspondence. I am also grateful to the Commonwealth Fund of New York which has supported this research in the form of a Harkness Fellowship.

2. Preliminaries. We begin this section by quoting the theorem of [12] which gives sufficient conditions for the continuity of module derivations on commutative separable Banach algebras.

For a Banach algebra $B$ and $n \in N, B^{n}$ denotes the ideal (not necessarily closed) spanned by $n$-fold products of elements of $B$.

THEOREM 1. Let $B$ be a commutative separable Banach algebra such that $B^{2}$ is of finite codimension in $B$ and which satisfies the following two conditions:

(1) there are no closed prime ideals of infinite codimension,

(2) every maximal ideal $M$ of $B$ has $M^{2}$ of finite codimension in $B$.

Then every module derivation from $B$ into a Banach-B-bimodule is continuous.

REMARKS. (a) Banach algebras for which every closed primary ideal is maximal are discussed in [17] and are there called $N^{*}$ algebras. A commutative completely regular $N^{*}$-algebra will clearly satisfy condition (1) and thus such algebras which also satisfy condition (2) (and the conditions on the whole algebra) will always have module derivations being continuous. Examples of algebras of this type are the Wiener algebra of functions with absolutely convergent Fourier series on $[0,1]$ and the algebra of continuous functions on $[0,1]$ of bounded variation as well as the more obvious example of continuous functions on a compact Hausdorff space. Note that there do exist commutative, unital, completely regular, semisimple, separable Banach algebras with nonmaximal proper closed prime ideals [3]. 
(b) W. G. Bade and P. C. Curtis, Jr. [1] have shown that, for a separable Banach algebra $B$ with unit, condition (2) is necessary and sufficient for the continuity of each homomorphism $\nu: B \rightarrow A$ where $A$ is a commutative Banach algebra with unit having a nontrivial finite dimensional radical.

As noted in [12] the conditions that $B^{2}$ is of finite codimension in $B$ and (2) are necessary in the sense that if either fails to hold then there exits a discontinuous module derivation from $B$ into some Banach- $B$-bimodule. So, from this point on, we assume that these conditions hold and we now examine the situation when condition (1) fails. This is carried out in $\S \S 3$ and 4 . Note that if $M$ is a maximal ideal in $B$ where $B$ is separable and $M^{2}$ is of finite codimension in $B$ then $M^{n}$ is closed and is of finite codimension in $B$ for all $n \in N$ (see [2]). Section 3 discusses the case where there exists a maximal ideal $M$ in $B$ such that the dimension of $M^{n} / M^{n+1}$ $\left(\equiv d\left(M^{n} / M^{n+1}\right)\right)$ is greater than or equal to 1 for all $n \in N$. Section 4 discusses the remaining possibility, i.e., for every maximal ideal $M$ in $B, M^{n}=M^{n+1}$ for some $n \in N$.

3. The case $d\left(M^{n} / M^{n+1}\right) \geqq 1$ for all $n \in N$. In $\S \S 3$ and 4 we are interested in Banach algebras where condition (1) of Theorem 1 fails and all the other conditions hold. In establishing the existence of discontinuous module derivations we may restrict our attention to Banach algebras which are also integral domains. For suppose condition (1) fails i.e., there is a closed prime ideal $K$ of infinite codimension in $B$; then $B / K$ is an integral domain and if there exists a discontinuous module derivation $\delta$ from $B / K$ into some Banach$B / K$-bimodule $X$ then there is a discontinuous module derivation $D$ from $B$ into the Banach-B-bimodule $X$. With $\pi$ denoting the natural map from $B \rightarrow B / K, D$ is given by $\delta * \pi$ and the module actions of $B$ on $X$ are given by $b \cdot x=\pi(b) \cdot x$ and $x \cdot b=x \cdot \pi(b)$ for $x$ in $X$ and $b$ in $B$. (By the same reasoning, to obtain a discontinuous module derivation from $B$ it is enough to to find one from some quotient algebra of $B$.) Also the other conditions of Theorem 1 on $B$ are carried to the quotient algebra $B / K$, i.e. if $d\left(B / B^{2}\right)<\infty$ and $d\left(M / M^{2}\right)<\infty$ for all maximal ideals $M$ of $B$ then $d\left((B / K) /(B / K)^{2}\right)<\infty$ and $d\left(N / N^{2}\right)<\infty$ for all maximal ideals $N$ of $B / K$. Thus, in short, in order to construct a discontinuous module derivation on a commutative separable Banach algebra $B$ where condition (1) of Theorem 1 fails but all the other conditions hold it is enough to construct one for the case where $B$ is also an integral domain.

In this section we restrict our attention to the case where there is a maximal ideal $M$ in $B$ such that $d\left(M^{n} / M^{n+1}\right) \geqq 1$ for all $n \in N$. 
Consider first the case where $d\left(M^{n} / M^{n+1}\right)=1$ for all $n \in N$. In this situation R. J. Loy [14] has proved that $B / \bigcap_{n=0}^{\infty} M^{n}$ is a Banach algebra of power series, i.e., a subalgebra $A$ of $C[[t]]$ which contains the polynomials and is a Banach algebra under a norm which makes the inclusion map $A \subseteq C[[t]]$ continuous. A class of examples of Banach algebras with a maximal ideal having this property are separable uniform algebras with an element $\phi$ of the maximal ideal space having a unique representing measure and a nontrivial Gleason part at $\phi$ [18]. More generally, it is not hard to see that, if $d\left(M^{n} / M^{n+1}\right)=C_{n}^{n+r-1}=(n+r-1) ! /(r-1) ! n !$ for all $n \in N$ and some integer $r \geqq 1$, then $B / \cap M^{n}$ is a Banach algebra of power series in $r$ commuting variables where such an algebra is given a similar definition to the case $r=1$. Now there exists a discontinuous module derivation from a Banach algebra of power series in $r$ variables $(r \geqq 1)$ and so for such $B$ we have a discontinuous module derivation. We do not prove this as it will follow from a more general theorem which we state below. First we establish some notation which we shall need in the proof of the theorem. For a domain $U$ in $C$, let $\mathscr{O}(U)$ denote the algebra of analytic functions on $U$ with the topology of uniform convergence on compact subsets of $U$. For an open subset $V$ of $C$ let $\mathcal{O}_{\vec{V}}$ be the inductive limit of the algebras $\mathcal{O}(U)$, with respect to the restriction maps, for $U$ an open neighbourhood of $\bar{V}$, i.e., $\mathscr{O}_{\bar{V}}$ is the algebra of germs of analytic functions on $\bar{V}$. For a unital algebra $A$, let $A^{-1}$ denote the group of units in $A$.

THEOREM 2. Let $B$ be a commutative Banach algebra with identity and let $\phi$ be an element of $\Phi_{B}$, the maximal ideal space of $B$, such that $1 \leqq d\left(M^{n} / M^{n+1}\right)<\infty$ for all $n \in N$, where $M=\operatorname{ker} \dot{\phi}$. Then there exists a discontinuous derivation from $B$ into some Banach-B-bimodule $X$.

Proof. By Theorem 4.1 of Read [16] $\Phi_{B}$ contains a nontrivial analytic variety at $\phi$ which is a neighborhood of $\phi$ in the metric topology. By an analytic variety at $\phi$ we mean the image of a oneto-one continuous mapping $F$ of a subvariety $V$ containing 0 of a domain in some $C^{m}$ where $F: V \rightarrow \Phi_{B}$ is such that $F(0)=\phi$ and for each $b$ in $B$ the map $\hat{b} \circ F$ is analytic on $V$. If $\left\{W_{1}, \cdots, W_{r}\right\}$ is a basis for $M / M^{2}$ then by Corollary 3.3 of [16] there are $s(\geqq 1)$ elements of this set (which we may take to be $W_{1}, \cdots, W_{s}$ ) such that, for each $n$, the set of homogeneous monomials of degree $n$ in $W_{1}, \cdots, W_{s}$ is linearly independent in $M^{n} / M^{n+1}$. Using this fact it is possible to construct a discontinuous module derivation in a similar fashion to the method used to construct one for the disc algebra in [4]. However we can deduce our conclusion more directly from the result 
in [4] as follows. First note that Read showed in Theorem 3.5 of [16] that the dimension $p$ of the variety $V$ at the origin is equal to $q+1$ where $q$ is the degree of the polynomial $\pi$ such that $\pi(n)=d\left(M^{n} / M^{n+1}\right)$ for all large $n$ (where we assign to the zero polynomial the degree -1$)$. Thus since $d\left(M^{n} / M^{n+1}\right) \geqq 1$ for all $n, q \geqq 0$ and so $p$ is nonzero. Thus the origin in a nonisolated point of $V$. We state the following nontrivial fact concerning analytic varieties: let $U$ be an open subset of $C^{m}$ and let $V$ be an analytic subvariety of $U$; then for each nonisolated point $s$ in $V$, there exists a oneto-one continuous map $G$ of the open unit disc $\Delta$ into $V$, with $G(0)=s$, such that $f \circ G$ is analytic in $\Delta$ whenever $f$ is analytic in $V$. We refer to Gunning and Rossi [10, Chapter 3] for the details of the proof. The basic idea is that, as a consequence of the Weierstrass preparation theorem $[10, p$. 68] the variety $V$ is locally like a Riemann surface. The result then follows by choosing suitable local parameters for this surface.

Returning to our argument we can thus embed the open unit disc $\Delta$ analytically in $\Phi_{B}$ at $\phi$, i.e., there exists $H: \Delta \rightarrow \Phi_{B}$ with $H(0)=\phi$ and $\hat{b} \circ H$ is analytic in $\Delta$ for all $b$ in $B$.

The next part of the argument is due to H. G. Dales and I am grateful for his permission to use it here. Take $b_{0}$ in $B$ with $\hat{b}_{0}(\phi)=0$ and $\hat{b}_{0} \circ H \neq 0$. This is clearly possible since $\Delta$ is embedded nontrivially in $\Phi_{B}$. Let $f_{0}=\hat{b}_{0} \circ H$ so that $f_{0}(0)=0$. Choose a neighbourhood $U$ of 0 such that $f_{0}$ has no zeros in $U \backslash\{0\}$. Then $f_{0}=z^{p} g_{0}$ for some $p \in N$ and some function $g_{0} \in \mathcal{O}(U)^{-1}$. Let $g_{1}=g_{0}^{1 / p}$ be defined on $U$ and put $f_{1}=z g_{1}$. Then $f_{1}$ is analytic on $U, f_{1}(0) \neq 0$ and $f_{1}^{\prime}(0) \neq 0$. Thus there exists an open neighbourhood $W$ of 0 such that $f_{1}: U \rightarrow W$ is one-to-one and onto (reducing $U$ if necessary). Let $h$ be the inverse of $f_{1}$ so that $h \in \mathcal{O}(W)$. By reducing $W$ if necessary we may assume that $h \in \mathcal{O}_{\bar{W}}$. Define $\theta: B \rightarrow \mathcal{O}_{\bar{W}}$ as follows: for each $b$ in $B$, put $\theta(b)=\hat{b} \circ H \circ h$. Then $\theta(b) \in \mathcal{O}_{\bar{W}}$ and $\theta: B-G_{\bar{W}}$ is a continuous homomorphism. Now $\theta\left(b_{0}\right)(s)=f_{0}(h(s))=\left[f_{1}(h(s))\right]^{p}=s^{p}$. So if $a_{0}=$ $\exp b_{0} \in B$ then $\theta\left(a_{0}\right)(s)=\exp \left(s^{p}\right)$. Clearly $E: s \rightarrow \exp \left(s^{p}\right)$ is transcendental with respect to the usual polynomials on $W$. Now applying the method of [4] as described in [20, p. 53-54] there exists a Banach space $X$ which is an $\mathcal{O}_{\bar{W}}$-module and a derivation $\delta$ from $\mathcal{O}_{\bar{W}}$ into $X$ that is zero on the polynomials on $W$ and $\delta(E) \neq 0$. (Briefly we choose a Banach space $X$ and a continuous linear operator $T$ on $X$ with spectrum contained in $W$, and regard $X$ as an $\mathcal{O}_{\bar{W}}$-module by $f \cdot x=f(T) x$ where $f(T)$ is given by the analytic functional calculus. We also choose $T$ to satisfy some additional restrictions as described on page 53 of [20]. Then we define $\delta$ to be zero on the polynomials on $W$ and by Theorem 8.7 of [20] we can extend $\delta$ to a derivation from $\mathcal{O}_{\bar{W}}$ into $X$ that is nonzero at $E$.) Thinking of $\theta$ mapping $B$ 
to $A(S)$ (where $A(S)$ is the set of analytic functions on $S$ that are continuous on $\bar{S}$ ) where $S$ is some disc, centre 0 , with $\bar{S} \subseteq W, X$ can be regarded as a Banach-B-bimodule $(b \cdot x=\theta(b) \cdot x$ etc. and $\|b \cdot x\|=\|\theta(b)(T) x\| \leqq K\|b\|\|x\|$ where $K$ is a constant, by the continuity of the single variable functional calculus). So $D=\delta \circ \theta$ is a module derivation from $B$ into $X$ with $D\left(b_{0}\right)=0$ but $D\left(a_{0}\right)=$ $D\left(\exp \left(b_{0}\right)\right) \neq 0$. Hence $D$ is discontinuous.

We note a corollary of the first part of the proof of Theorem 2 which relates the finiteness of $d\left(M^{n} / M^{n+1}\right)$ for all $n \in N$ for some maximal ideal $M$ in $B$ with the failure of condition (1) of Theorem 1 .

COROLlaRY 3. Let $B$ be a commutative Banach algebra with identity and let $\phi \in \Phi_{B}$ with $1 \leqq d\left(M^{n} / M^{n+1}\right)<\infty$ for all $n \in N$, where $M=\operatorname{ker} \phi$. Then $B$ contains a closed prime ideal of infinite codimension.

Proof. As in the first part of the proof of Theorem 2 we have a continuous one-to-one map $H: \Delta \rightarrow \Phi_{B}$ with $H(0)=\phi$ and $\hat{b} \circ H$ analytic in $\Delta$ for each $b$ in $B$. Let $K=\bigcap_{\psi \in H(\Delta)} M_{\psi}$ where $M_{\psi}=\operatorname{ker} \psi$. Then $K$ is prime since if $f g \in K$ then $\hat{f} \hat{g} \circ H=0$ on $\Delta$ and so $(\hat{f} \circ H)(\hat{g} \circ H)=0$ on $\Delta$. Thus either $\hat{f} \circ H=0$ or $\hat{g} \circ H=0$ on $\Delta$ (since both $\hat{f} \circ H$ and $\hat{g} \circ H$ are analytic) i.e., either $f \in K$ or $g \in K$. Since $H$ is one-to-one it is clear that $K$ has infinite codimension.

REMARKS. In $\S 4$ we give an example of a commutative Banach algebra which is an integral domain for which there exists only one maximal ideal $M$ and $d\left(M / M^{2}\right)=1$ and $d\left(M^{n} / M^{n+1}\right)=0$ for $n>1$. This shows that the converse of Corollary 3 is not true. Negating Corollary 3 shows that if every closed prime ideal of $B$ is maximal then, for every maximal ideal $M$ of $B, d\left(M^{n} / M^{n+1}\right) \in\{0, \infty\}$ for some $n \in N$. The Banach algebra $C^{n}[0,1]$ shows that the infinite case is possible (see [17, p. 300]). It would be of interest to know whether by strengthening the hypothesis that every closed prime ideal is maximal we can make it impossible for the infinite case to occur. For example, a possible question might be: if $B$ is completely regular and if $J_{\phi}$, given by $J_{\phi}=\left\{f \in B: \hat{f}\right.$ vanishes on a neighbourhood of $\phi$ in $\left.\Phi_{B}\right\}$, satisfies $\bar{J}_{\phi}=M_{\phi} \equiv \operatorname{ker} \phi$ for all $\phi \in \Phi_{B}$ is $d\left(M_{\phi} / M_{\phi}^{2}\right)<\infty$ for all $\phi$ in $\Phi_{B}$ ?

Note that the "non-appearance" of closures in the hypothesis of Theorem 2 is crucial for the proof. Indeed S. J. Sidney [19, Example 5.18] has constructed a Swiss cheese algebra whose maximal ideal space contains an element $\phi$ such that $d\left(\left(M_{\phi}^{n}\right)^{-} /\left(M_{\phi}^{n+1}\right)^{-}\right)=1$ for each $n$, where $M=\operatorname{ker} \phi$. Clearly the maximal ideal space has no analytic structure of any kind. 
Theorem 2 yields little indication of how the discontinuous derivation operates on the Banach algebra $B$. In particular examples, however, it is easy to glean some more information. In [4] H. G. Dales constructed a discontinuous module derivation from the disc algebra, $A(\Delta)$, which is zero on the polynomials in $z$ and nonzero at $\exp z$, for example. An immediate consequence of this is the existence of a discontinuous module derivation from $A(B)$ and $A\left(\Delta^{n}\right)$ (where $A(B)$ and $A\left(\Delta^{n}\right)$ are the algebras of analytic functions on the open unit ball and the open polydisc in $C^{n}$, resp. which are continuous on $\bar{B}$ and $\bar{d}^{n}$, resp.) which is zero on the polynomials in the coordinate functions, $z_{1}, \cdots, z_{n}$, and nonzero at $\exp z_{i}$ for some $i$. In fact we can say more.

Proposition 4. There exists a discontinuous module derivation from both $A(B)$ and. $A\left(\Delta^{n}\right)$ which is zero on the polynomials in $z_{1}, \cdots, z_{n}$ and nonzero at $\exp z_{i}$ for all $i, 1 \leqq i \leqq n$, in each case.

Proof. Let $v=\left(v_{1}, \cdots, v_{n}\right) \in \partial B$ be such that $v_{i} \neq 0$ for all $i$. We imbed $\Delta$ in $\Phi_{A(B)}$ by the following map: $H(\lambda)=\lambda v,(\lambda \in \Delta)$. If $\Omega$ is the closed disc, centred at 0 , of radius $1 / 2$, say, then by [4] there exists a discontinuous module derivation $\delta$ from $A(\Omega)$ which is zero on the polynomials in $z$ and nonzero at $\exp z$. Define $\theta: B \rightarrow A(\Omega)$ by $\theta(b)=\hat{b} \circ H$ and, as at the end of the proof of Theorem 2, put $D=\delta \circ \theta$. Notice that $\theta\left(z_{j} / v_{j}\right)(z)=z$ for $z \in \Omega$ and $1 \leqq j \leqq n$, and so $D$ is zero on the polynomials in $z_{1}, \cdots, z_{n}$ but is nonzero at $\exp z_{i}$ for $1 \leqq i \leqq n$.

The proof for $A\left(\Delta^{n}\right)$ follows in a similar fashion replacing the map $H$ by the map $G: \Delta \rightarrow \Phi_{A\left(\Delta^{n}\right)}$ given by $G(\lambda)=(\lambda, \lambda, \cdots, \lambda)$.

Let $H^{\circ}(\Delta)$ be the Banach algebra of bounded analytic functions on $\Delta$. Let $\mathscr{P}$ be the subalgebra of $\mathscr{O}_{\bar{W}}$ of polynomials in one variable. We have the following result which contains in its proof a proof of the fact that a function $f$ in $H^{\circ}(\Delta) \backslash A(\Delta)$ cannot satisfy an equation of the form:

$$
p_{m}(z)[f(z)]^{m}+\cdots+p_{1}(z) f(z)+p_{0}(z)=0 \quad(z \in \Delta),
$$

where $p_{j}$ is a polynomial, $1 \leqq j \leqq m$. This fact may be interesting in its own right.

Proposition 5. There exists a discontinuous module derivation from $H^{\infty}(\Delta)$ which is zero on a dense subset of $H^{\infty}(\Delta)$.

Proof. Let $\gamma: H^{\infty}(\Delta) \rightarrow \mathcal{O}_{\bar{\Delta}}$ be defined by $(\gamma f)(z)=f(z / 2)$ for all $z$ in $2 \Delta$ and all $f$ in $H^{\circ}(\Delta)$. Then $\gamma$ is a monomorphism from $H^{\infty}(\Delta)$ 
into $\mathscr{O}_{\bar{\Delta}}$ and $\gamma\left(H^{\infty}(\Delta)\right)$ contains an element of $\mathscr{O}_{\overline{3}}$ that is transcendental over $\mathscr{P}^{\circ}$ e.g., $\exp z$.

Let $f \in H^{\circ}(\Delta) \backslash A(\Delta)$; we claim that $f$ cannot satisfy an equation of the form $(*)$. For suppose $f$ does satisfy such an equation. Taking radial limits we have $p_{m}(z)[f(z)]^{m}+\cdots+p_{0}(z)=0$ for $z \in\{z \in C:|z|=1\}=\mathscr{T}$, where we use $f$ to represent the function on $\mathscr{T}$ given by $f(z)=\lim _{r \rightarrow 1} f(r z)$. Now, by hypothesis, $f$ has some point of discontinuity on $\mathscr{T}$, say $\lambda$. Let $C_{r}$ be the set of cluster points of $f(z)$ as $z \rightarrow \lambda$ from the 'right' on $\mathscr{T}$, and $C_{l}$ the set of cluster points of $f(z)$ as $z \rightarrow \lambda$ from the 'left' on $\mathscr{T}$. Note that since $f$ satisfies $(*) f\left(\lambda_{k}\right) \nrightarrow \infty$ as $\lambda_{k} \rightarrow \lambda$ for any sequence $\left\{\lambda_{k}\right\}$ in $\mathscr{T}$. $C=C_{r} \cup C_{l}$ must be infinite; for suppose otherwise, i.e., $C_{r}=\left\{\mu_{1}, \cdots, \mu_{s}\right\}$, $C_{l}=\left\{\eta_{1}, \cdots, \eta_{t}\right\}$. Choose a polynomial $P$ so that $P\left(\mu_{1}\right)=\cdots=$ $P\left(\mu_{s}\right)=0$ and $P\left(\eta_{1}\right)=\cdots=P\left(\eta_{t}\right)=1$. Then $h(z)=P(f(z))$, when extended to the disc, is in $H^{\circ}(\Delta)$. But as $z \rightarrow \lambda$ from the 'right' $h(z) \rightarrow 0$ and as $z \rightarrow \lambda$ from the 'left' $h(z) \rightarrow 1$, i.e., $h$ has a simple 'jump' discontinuity which is impossible for an $H^{\circ}(\Delta)$ function extended to $\mathscr{T}$. Hence $C$ is infinite. We may assume that $(z-\lambda)$ is not a factor of all the polynomials $p_{j}, 1 \leqq j \leqq m$. Consider the equation in $w: p_{m}(\lambda) w^{m}+\cdots+p_{1}(\lambda) w+p_{0}(\lambda)=0$. Not all the coefficients are zero. However each point of $C$ is a root of this equation. Thus we have a contradiction and so $f$ cannot satisfy an equation of the form (*). It follows from this that for any $f \in H^{\infty}(\Delta) \backslash A(\Delta)$, $\gamma(f)$ is transcendental over $\mathscr{P}$. As before it follows from Theorem 8.7 of [20] that there is a derivation $\delta$ from $O_{\text {; }}$ to an $\sigma_{\text {-module }} X$ which is zero on $\mathscr{P}$ and may be arbitrarily defined on any subset of $\mathscr{O}_{\overline{4}}$ containing elements transcendental over $\mathscr{F}$. Choose $\delta$ so that $\delta$ is zero on $\mathscr{P}$ and on $\gamma\left(H^{\infty}(\Delta) \backslash A(\Delta)\right)$ but is nonzero at $\gamma(\exp z)$. As before we can make $X$ into a Banach- $H^{\circ}(\Delta)$-bimodule using the single variable functional calculus. Then $D=\delta \circ \gamma$ is a discontinuous module derivation from $H^{\circ}(\Delta)$ into $X$ that is zero on the polynomials and on $H^{\infty}(\Delta) \backslash A(\Delta)$. Hence $D$ is zero on a dense subset of $H^{\infty}(\Delta)$.

With regard to the module derivation $D$ constructed in Theorem 2 it is clear from the ideas used in the proof of Proposition 5 that $D$ need not, in general, be zero on a dense subset of $B$ (e.g., in the proof of Proposition 5 we can choose $\delta$ arbitrarily on $\gamma\left(H^{\infty}(\Delta) \backslash A(\Delta)\right)$ and so we could choose it to be nonzero everywhere there). However, in the notation of the proof of Theorem $2, D$ is a nonzero module derivation on the Banach subalgebra $A$ generated by the element $b_{0}$ and the identity, and $D$ is zero on a dense subset of $A$.

4. The case $d\left(M^{n} / M^{n+1}\right)=0$ for some $n \in N$ for every maximal ideal $M$. As before let $B$ be a commutative separable Banach algebra which is an integral domain such that $d\left(B / B^{2}\right)<\infty$ and 
$d\left(M / M^{2}\right)<\infty$ for all maximal ideals $M$ in $B$. We have now shown that there exists a discontinuous module derivation in every case except when $d\left(M^{n} / M^{n+1}\right)=0$ for some $n \in N$ for every maximal ideal $M$. If $d\left(M^{n} / M^{n+1}\right)=0$ put $A=M^{n}$. As noted previously, separability implies that $A$ is closed, and clearly $A$ is an integral domain and $A^{2}=A$. Thus it seems reasonable at first to restrict our attention to integral domains $B$ where $B^{2}=B$. If we further suppose that $B$ is radical then $B \oplus C 1$ (where 1 is a unit for $B$ ) gives us the simplest example of the type of behaviour we are interested in. The typical example of this sort of algebra is the weighted convolution algebra, $L^{1}\left(\boldsymbol{R}^{+}, w\right)$, on the half-line, i.e., $L^{1}\left(\boldsymbol{R}^{+}, w\right)$ is the space of complex measurable functions $f$ on $\boldsymbol{R}^{+}$such that $\|f\|=$ $\int_{0}^{\infty}|f(t)| w(t) d t<\infty$ where $w: \boldsymbol{R}^{+} \rightarrow \boldsymbol{R}^{+} \backslash\{0\}$ is a continuous weight function with $w(0)=1$ and $w(x+y) \leqq w(x) w(y)$. Multiplication is given by convolution: $(f * g)(t)=\int_{0}^{t} f(s) g(t-s) d s$. By choosing $w$ carefully we can make $L^{1}\left(\boldsymbol{R}^{+}, w\right)$ a radical Banach algebra which is an integral domain and which has a bounded approximate identity (see [7]). We have been unable to settle the question of the continuity of module derivations from this algebra and so the problem remains: with $L^{1}\left(\boldsymbol{R}^{+}, w\right)$ as above, is there a discontinuous module derivation from $L^{1}\left(\boldsymbol{R}^{+}, w\right)$ ? Classifying the closed ideals of $L^{1}\left(\boldsymbol{R}^{+}, w\right)$ is a well-known open problem and even if we could do this it is still not clear whether every module derivation is continuous. The problem seems to require some new techniques. It follows from [13] that every derivation: $L^{1}\left(\boldsymbol{R}^{+}, w\right) \rightarrow L^{1}\left(\boldsymbol{R}^{+}, w\right)$ is continuous. Another area of study here is to decide how typical $L^{1}\left(\boldsymbol{R}^{+}, w\right)$ is amongst radical Banach algebras with bounded approximate identities. A specific task might be to find a radical Banach algebra with a bounded approximate identity which is not isomorphic to a quotient of $L^{1}\left(\boldsymbol{R}^{+}, w\right)$ by some closed ideal. Recent results on algebras with bounded approximate identities which may be related to this question can be found in [21].

We now give an example of a Banach algebra which is an integral domain for which $d\left(M^{n} / M^{n+1}\right)<\infty$ for all $n \in \boldsymbol{N}$ for every maximal ideal $M$ but which has a maximal ideal $N$ such that $d\left(N / N^{2}\right)=1$ and $d\left(N^{n} / N^{n+1}\right)=0$ for $n>1$. In other words, the chain of descending ideals of powers of $N$ starts nontrivially but then stops. It is not hard to extend the idea of the example to construct a similar Banach algebra which is an integral domain for which $d\left(N^{n} / N^{n+1}\right) \neq 0$ for $1 \leqq n \leqq k$ and $d\left(N^{n} / N^{n+1}\right)=0$ for $n>k$ (where $k$ is some integer $\geqq 1$ ). In [16, Example 5.2] Read describes a rather complicated uniform algebra $A$ with a maximal ideal $N$ such that $d\left(N^{n} / N^{n+1}\right)=1$ for $1 \leqq n \leqq k$ and $N^{k+1}=N^{k+2}$. This algebra, 
however, is constructed as the closure of the sum of two algebras each of which are algebraic tensor products of smaller algebras and so it is clear that $A$ is not an integral domain. It is the fact that the following simple example is an integral domain which makes it more interesting in our context.

EXAMPLe. Let $A=L^{1}\left(\boldsymbol{R}^{+}, w\right)$ as above with $w$ chosen so that $A$ is radical, is an integral domain, and has a bounded approximate identity. By [11] there exists a positive Borel measure $\mu$ on $\boldsymbol{R}^{+}$ which is singular (w.r.t. Lebesgue measure on $\boldsymbol{R}^{+}$) such that $\mu * \mu$ is absolutely continuous w.r.t. Lebesgue measure. We can choose $\mu$ to have compact support. Let $B=C 1 \oplus C \mu \oplus A$ where 1 is an identity for $B$ and all the other multiplications are given by convolution. Note that $\mu * f$ is in $A$ for all $f \in A$. Also $d\left(B / B^{2}\right)=1$ and the only maximal ideal in $B$ is $C \mu \oplus A=N$, say. $B$ is an integral domain by the Titchmarsh convolution theorem extended to measures. Finally $d\left(N / N^{2}\right)=1$ but $d\left(N^{n} / N^{n+1}\right)=0$ for $n>1$.

We conclude by describing an example due to $H$. G. Dales which shows that there exist Banach algebras $B$ with $d\left(M / M^{2}\right) \leqq k(M)<\infty$ for all maximal ideals $M$ where $k(M)$ has no upper bound.

EXAMPLE. Let $\bar{\Delta}^{n}$ be the closed unit polydisc in $C^{n}$ and let $A_{n}$ be the polydisc algebra, $A\left(\Delta^{n}\right)$, defined earlier. Let $C^{\infty}=\left\{z=\left(z_{0}, z_{1}, \cdots\right)\right.$ : $z_{i} \in C$ for all $\left.i \geqq 0\right\}$. Let $\widetilde{\Delta}^{n}=\left\{z: z_{0}=1 / n\right.$, and, $\left|z_{1}\right| \leqq 1 / n, \cdots,\left|z_{n}\right| \leqq$ $\left.1 / n, z_{n+1}=z_{n+2}=\cdots=0\right\} \subseteq C^{\infty}$, so that $\widetilde{d}^{n}$ is a copy of $d^{n}$. Thus we can regard $A_{n}$ as an algebra of functions on $\widetilde{\Delta}^{n}$. Let

$$
X=\bigcup_{n=1}^{\infty} \widetilde{\Delta}^{n} \cup\{(0,0, \cdots)\} \text {. }
$$

$X$ is a compact subset of $C^{\infty}$. Let $B=\left\{f \in C(X): f_{\mid \widetilde{I}_{n}} \in A_{n}\right\}$. It is easy to see that the maximal ideal space of $B$ is $X$. If $M$ is the maximal ideal associated with $(0,0, \cdots)$ then $M^{2}=M$ since $M$ has a bounded approximate identity. Every other maximal ideal corresponds to a maximal ideal of $A_{n}$ so that $d\left(M / M^{2}\right)<\infty$ for all maximal ideals $M$ of $B$. However if $M_{n}=\{f: f(1 / n, 0,0, \cdots)=0\}$ then $d\left(M_{n} / M_{n}^{2}\right)=n$. Hence there does not exist a constant $K$ such that $d\left(M / M^{2}\right) \leqq K$ for all maximal ideals $M$.

\section{REFERENCES}

1. W. G. Bade and P. C. Curtis, Jr., Prime ideals and automatic continuity problems for Banach algebras, (to appear in J. Functional Analysis).

2. J. P. R. Christensen, Codimension of some subspaces in a Fréchet algebra, Proc. Amer. Math. Soc., 57 (1976), 276-278.

3. J. M. Cusack, Homomorphisms and derivations on Banach algebras, $\mathrm{Ph}$. D. thesis, 
University of Edinburgh, 1976.

4. H. G. Dales, The uniqueness of the functional calculus, Proc. London Math. Soc., (3) 27 (1973), 638-648.

5. - A discontinuous homomorphism from $C(X)$, (to appear in Amer. J. Math.).

6. - Discontinuous homomorphisms from topological algebras, (preprint).

7. P. G. Dixon, Semi-prime Banach algebras, J. London Math. Soc., (2) 6 (1973), 676-678.

8. J. Esterle, Injections de semi-groups divisibles dans des algèbres de convolution et construction d'homomorphismes discontinus de $C(K)$ ), (to appear in Proc. London Math. Soc.).

9. J. Este:le, (preprint).

10. R. Gunning and H. Rossi, Analytic Functions of Several Complex Variables, PrenticeHall, Englewood Cliffs, N. J., 1965.

11. E. Hewitt and H. S. Zuckerman, Singular measures with absolutely continuous convolution souares, Proc. Cambridge Philos. Soc., 62 (1966), 399-420.

12. N. P. Jewell, The continuity of module and higher derivations, (to appear in Pacific J. Math.).

13. N. P. Jewell and A. M. Sinclair, Epimorphisms and derivations on $L^{1}[0,1]$ are continuous, Bull. London Math. Soc., 8 (1976), 135-139.

14. R. J. Loy, Banach algebras of power series, J. Aust. Math. Soc., 17 (1974), 263-273. 15. Commutative Banach algebras with non-unique complete norm topology, Bull. Austral. Math. Soc., 10 (1974), 409-420.

16. T. T. Read, The powers of a maximal ideal in a Banach algebra and analytic structure, Trans. Amer. Math. Soc., 161 (1971), 235-248.

17. C. E. Rickart, General Theory of Banach Algebras, R. E. Krieger, New York, 1974. 18. S. J. Sidney, Point derivations in certain sup-norm algebras, Trans. Amer. Math. Soc., 131 (1968), 119-127.

19. - Properties of the sequence of closed powers of a maximal ideal in a supnorm algebra, Trans. Amer. Math. Soc., 131 (1968), 128-148.

20. A. M. Sinclair, Automatic continuity of linear operators, London Math. Soc. Lecture Note Series 21 (1976).

21. - Bounded approximate identities, factorization, and a convolution algebra, (preprint).

Received December 9, 1976 and in revised form March 7, 1977.

UNIVERSity OF CALIFORNIA

BERKELEY, CA 94720 



\section{PACIFIC JOURNAL OF MATHEMATICS}

\section{EDITORS}

RICHARD ARENS (Managing Editor)

University of California

Los Angeles, CA 90024

Charles W. Curtis

University of Oregon

Eugene, OR 97403

C. C. MOORE

University of California

Berkeley, CA 94720

\section{J. DugundJI}

Department of Mathematics

University of Southern California

Los Angeles, CA 90007

R. FinN and J. Milgram

Stanford University

Stanford, CA 94305

ASSOCIATE EDITORS
E. F. BECKENBACH
B. H. NeumanN
F. WOLF
K. YOSHIDA

\section{SUPPORTING INSTITUTIONS}

UNIVERSITY OF BRITISH COLUMBIA CALIFORNIA INSTITUTE OF TECHNOLOGY

UNIVERSITY OF CALIFORNIA

MONTANA STATE UNIVERSITY

UNIVERSITY OF NEVADA, RENO

NEW MEXICO STATE UNIVERSITY

OREGON STATE UNIVERSITY

UNIVERSITY OF OREGON

OSAKA UNIVERSITY

\author{
UNIVERSITY OF SOUTHERN CALIFORNIA \\ STANFORD UNIVERSITY \\ UNIVERSITY OF HAWAII \\ UNIVERSITY OF TOKYO \\ UNIVERSITY OF UTAH \\ WASHINGTON STATE UNIVERSITY \\ UNIVERSITY OF WASHINGTON \\ AMERICAN MATHEMATICAL SOCIETY
}

The Supporting Institutions listed above contribute to the cost of publication of this Journal, but they are not owners or publishers and have no responsibility for its content or policies.

Mathematical papers intended for publication in the Pacific Jaurnal of Mathematics should be in typed form or offset-reproduced, (not dittoed), double spaced with large margins. Please do not use built up fractions in the text of your manuscript. You may however, use them in the displayed equations. Underline Greek letters in red, German in green, and script in blue. The first paragraph or two must be capable of being used separately as a synopsis of the entire paper. Items of the bibliography should not be cited there unless absolutely necessary, in which case they must be identified by author and Journal, rather than by item number. Manuscripts, in triplicate, may be sent to any one of the editors. Please classify according to the scheme of Math. Reviews, Index to Vol. 39. All other communications should be addressed to the managing editor, or Elaine Barth, University of California, Los Angeles, California, 90024.

The Pacific Journal of Mathematics expects the author's institution to pay page charges, and reserves the right to delay publication for nonpayment of charges in case of financial emergency.

100 reprints are provided free for each article, only if page charges have been substantially paid. Additional copies may be obtained at cost in multiples of 50 .

The Pacific Journal of Mathematics is issued monthly as of January 1966. Regular subscription rate: $\$ 7200$ a year (6 Vols., 12 issues). Special rate: $\$ 36.00$ a year to individual members of supporting institutions.

Subscriptions, orders for back numbers, and changes of address should be sent to Pacific Journal of Mathematics, 103 Highland Boulevard, Berkeley, California, 94708.

PUBLISHED BY PACIFIC JOURNAL OF MATHEMATICS, A NON-PROFIT CORPORATION

Printed at Kokusai Bunken Insatsusha (International Academic Printing Co., Ltd.). 8-8, 3-chome, Takadanobaba, Shinjuku-ku, Tokyo 160, Japan.

Copyright (C) 1975 by Pacific Journal of Mathematics Manufactured and first issued in Japan 


\section{Pacific Journal of Mathematics}

\section{Vol. 71, No. $2 \quad$ December, 1977}

Krishnaswami Alladi and Paul Erdős, On an additive arithmetic

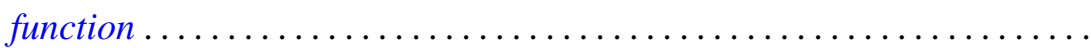

James Bailey and Dale Rolfsen, An unexpected surgery construction of a

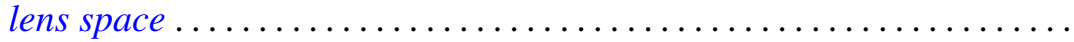

Lawrence James Brenton, On the Riemann-Roch equation for singular

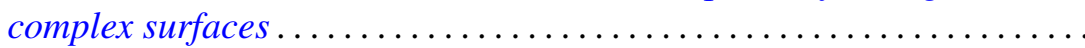

James Glenn Brookshear, Projective ideals in rings of continuous

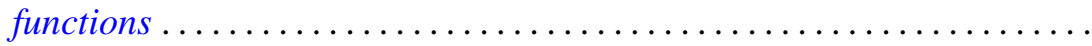

Lawrence Gerald Brown, Stable isomorphism of hereditary subalgebras of

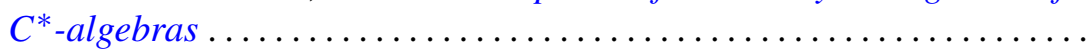

Lawrence Gerald Brown, Philip Palmer Green and Marc Aristide Rieffel, Stable isomorphism and strong Morita equivalence of $C^{*}$-algebras....

N. Burgoyne, Robert L. Griess, Jr. and Richard Lyons, Maximal subgroups

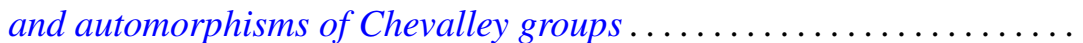
365

Yuen-Kwok Chan, Constructive foundations of potential theory .... 405

Peter Fletcher and William Lindgren, On $w \Delta$-spaces, $w \sigma$-spaces and

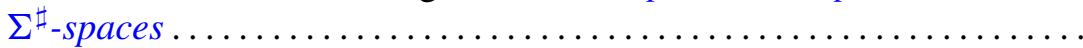

Louis M. Friedler and Dix Hayes Pettey, Inverse limits and mappings of

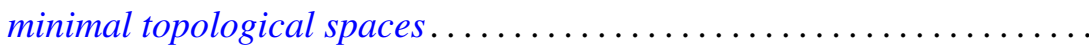

Robert E. Hartwig and Jiang Luh, A note on the group structure of unit regular ring elements.

I. Martin (Irving) Isaacs, Real representations of groups with a single involution ...

Nicolas P. Jewell, The existence of discontinuous module derivations . .

Antonio M. Lopez, The maximal right quotient semigroup of a strong semilattice of semigroups .......................

Dennis McGavran, $T^{n}$-actions on simply connected $(n+2)$-manifolds

Charles Anthony Micchelli and Allan Pinkus, Total positivity and the exact $n$-width of certain sets in $L^{1}$.

Barada K. Ray and Billy E. Rhoades, Fixed point-theorems for mappings with a contractive iterate .......................

Fred Richman and Elbert A. Walker, Ext in pre-Abelian categories. .

Raymond Craig Roan, Weak* generators of $H^{\infty}$ and $l^{1}$..

Saburou Saitoh, The exact Bergman kernel and the kernels of Szegö type...

Kung-Wei Yang, Operators invertible modulo the weakly compact 